\title{
Fine mapping of the chromosome 2p12-16 dyslexia susceptibility locus: quantitative association analysis and positional candidate genes SEMA4F and OTX1
}

\author{
Clyde Francks ${ }^{a}$, Simon E. Fisher ${ }^{\mathrm{a}}$, Richard K. Olson ${ }^{\mathrm{b}}$, Bruce F. Pennington ${ }^{\mathrm{c}}$, Shelley D. \\ Smith $^{d}$, John C. DeFries ${ }^{b}$ and Anthony P. Monaco ${ }^{a}$ \\ ${ }^{a}$ Wellcome Trust Centre for Human Genetics, University of Oxford, UK; ${ }^{\mathrm{b}}$ Institute for Behavioral Genetics, \\ University of Colorado, Boulder, Colorado; ' Department of Psychology, University of Denver, Denver, \\ Colorado; 'Center for Human Molecular Genetics, University of Nebraska Medical Center, Omaha, \\ Nebraska, USA
}

Correspondence to A.P. Monaco, Wellcome Trust Centre for Human Genetics, University of Oxford, Roosevelt Drive, Oxford, UK. Tel: +44 1865 287502; fax: +44 1865 287650; E-mail:

anthony.monaco@well.ox.ac.uk

Received 10 December 2001 accepted 28 December 2001

\begin{abstract}
A locus on chromosome 2p12-16 has been implicated in dyslexia susceptibility by two independent linkage studies, including our own study of 119 nuclear twin-based families, each with at least one reading-disabled child. Nonetheless, no variant of any gene has been reported to show association with dyslexia, and no consistent clinical evidence exists to identify candidate genes with any strong a priori logic. We used 21 microsatellite markers spanning 2p12-16 to refine our 1-LOD unit linkage support interval to $12 \mathrm{cM}$ between D2S337 and D2S286. Then, in quantitative association analysis, two microsatellites yielded $P$ values $<0.05$ across a range of reading-related measures (D2S2378 and D2S2114). The exon/intron borders of two positional candidate genes within the region were characterized, and the exons were screened for polymorphisms. The genes were Semaphorin4F (SEMA4F), which encodes a protein involved in axonal growth cone guidance, and $O T X 1$, encoding a homeodomain transcription factor involved in forebrain development. Two non-synonymous single nucleotide polymorphisms were found in SEMA4F, each with a heterozygosity of 0.03 . One intronic single nucleotide polymorphism between exons 12 and 13 of SEMA4F was tested for quantitative association, but no significant association was found. Only one single nucleotide polymorphism was found in OTX1, which was exonic but silent. Our data therefore suggest that linkage with reading disability at 2p12-16 is not caused by coding variants of SEMA4F or OTX1. Our study outlines the approach necessary for the identification of genetic variants causing dyslexia susceptibility in an epidemiological population of dyslexics. Psychiatr Genet 12:35-41 (C) 2002 Lippincott Williams \& Wilkins
\end{abstract}

Keywords: linkage disequilibrium, quantitative trait, dyslexia, microsatellite repeats, polymorphism, single nucleotide

\section{INTRODUCTION}

Dyslexia is a common neurological syndrome that manifests most obviously as a disorder of reading (Smith et al., 1996). The syndrome has a strong but complex genetic component (DeFries et al., 1987; Smith et al., 1996; Olson et al., 1999; Fisher et al., in press), and evidence for susceptibility loci on chromosomes 1, 2, 3, 6, 15 and 18 has been reported (Fisher \& Smith, 2001; Nopola-Hemmi et al., 2001; Fisher et al., 2002). Two independent genome-wide linkage studies, using very different analytical approaches, have provided convergent evidence that a dyslexia susceptibility locus maps to chromosome 2p12-16 (Fagerheim et al., 1999; Fisher et al., 2002). Fagerheim et al. (1999) analyzed a single fourgeneration pedigree with a qualitatively defined affection status, and reported a non-parametric linkage $(P=0.0009)$ for a region of $2 \mathrm{p} 15-16$, which was the strongest linkage in that genome screen. Fisher et al. (2002) analyzed a sample of 119 nuclear sib-pair families drawn from the Colorado twin study of reading disability (DeFries et al., 1987; Olson et al., 1999), and tested for linkage directly to quantitative reading-related traits. Using one analytical approach based on partitioning of trait variance components, a quantitative trait locus (QTL) on 2p12-16 again provided the strongest evidence for 
linkage in the genome $(P=0.0008$ with a wordrecognition measure). The present study was performed using the latter sample of 119 Colorado families. The robust detection of the 2p12-16 QTL by the linkage studies of both Fagerheim et al. (1999) and Fisher et al. (2002) suggests that the locus is likely to have an important effect in a broad population of dyslexics.

According to Genemap99 (website: http:// www.ncbi.nlm.nih.gov/genemap99/), the 2p12-16 region contains 68 known genes, plus approximately 250 uncharacterized messenger RNAs and expressed sequence tags. To refine linkage mapping of the 2 p1216 QTL, we genotyped 21 microsatellite markers spanning the locus with an average intermarker interval of $1.6 \mathrm{cM}$. We then performed a recently implemented quantitative sib-pair association analysis (Abecasis et al., 2000) with the 21 microsatellites and four correlated measures of reading disability. Association analysis has the potential to provide greater positional resolution than linkage analysis, since the extent of linkage disequilibrium (LD) reflects many more ancestral meioses (Hartl and Clark, 2001). In addition, the quantitative analytical approach is expected to be more powerful than analysis based on qualitative diagnostic schemes, and to better accommodate the phenotypic and genetic complexity of dyslexia (Cardon et al., 1994, 1995; Fisher et al., 1999; Marlow et al., 2001).

We then decided on a candidate gene screening approach within the region of linkage rather than a higher density LD mapping approach (e.g. using a 5$50 \mathrm{~kb}$ intermarker interval) due to the prohibitive number of markers needed to span the locus and the inherent multiple testing problem with this latter approach. The aim was to identify potentially functional variants within candidate genes that could be tested directly for association. Since there are no a priori candidate genes for dyslexia, we selected two positional candidates for study on the basis of their reported functions and patterns of expression. These were Semaphorin4F (SEMA4F) and OTX1.

SEMA4F (SEMAM, SEMAW; OMIM 603706) belongs to the type IV family of membrane-bound semaphorins, which are a family of secreted and membrane-bound proteins that regulate axonal and dendritic growth and guidance (Kolodkin et al., 1993; Nakamura and Kalb, 2000). SEMA4F causes the collapse of retinal ganglion cell growth cones in vitro, and is expressed in rat embryonic spinal column, retinal ganglion cells and around the optic nerve, and at high levels in the adult rat central nervous system (Encinas et al., 1999). The expression pattern and functional profile of SEMA4F therefore made it a good candidate for the 2p12-16 QTL, especially since subtle defects of the visual system have been reportedly associated with dyslexia (Eden et al., 1996).

OTX1 (OMIM 600036) encodes a homeoboxcontaining transcription factor, which is involved in forebrain morphogenesis and the differentiation of neural structures, especially of the cortex (Acampora et al., 1999). OTX1 is also postnatally expressed in the pituitary gland and/or the hypothalamus (Acampora et al., 1998). Mice lacking OTX1 show seizures and brain abnormalities (Acampora et al., 1996), and also transient dwarfism and hypogonadism (Acampora et al., 1998), suggesting an involvement in the prepubescent control of some major developmental and reproductive hormones. In this respect, OTX1 might relate to a proposed link between foetal hormone levels and reading disability (Smith et al., 1996). More generally, the involvement of OTX1 in brain patterning and corticogenesis made it a good candidate gene for dyslexia.

\section{MATERIALS AND METHODS}

The family sample

One hundred and nineteen nuclear twin-based families were drawn from an ongoing study by the Colorado Learning Disabilities Research Centre (DeFries et al., 1987; Olson et al., 1999). School records from 27 Colorado districts were used to identify twins. Parental permission was then sought to examine the twins' files for evidence of reading problems. If at least one member of a twin pair showed a positive school history of reading problems, both twins and any other siblings were invited to the University of Colorado where they were administered a battery of psychometric tests. The standardized test scores were used directly for quantitative linkage analysis. Details of tests are presented in Olson et al. (1999) and Fisher et al. (2002). Monozygotic twin pairs were excluded from the present study sample, although if additional sibs were available for a $\mathrm{MZ}$ pair, one of the $\mathrm{MZ}$ pair and any additional non-twin siblings could be included. The 119 families yielded 180 total pairs. The mean age for the twins and siblings was 11.6 years (range, 8-19 years).

\section{Microsatellite markers}

Microsatellites were assigned genetic positions in Haldane centimorgans based on data from Genethon (website: http://www.genethon.fr/), the Cooperative Human Linkage Centre maps (v2hd.abi.sexave, v8c8.recmin.v1.abi.sexave, Recombination-Min; 
website: http://www.lpg.nci.nih.gov/CHLC/), and the Marshfield genetic maps (website: http://www.marshmed.org/genetics/contents.htm). The relative positions of markers (Fig. 1) were verified using published physical mapping data (Whitehead contigs 2.4, 2.5; website: http://www-genome.wi.mit.edu/; Resch et al., 1998; Kirschner et al., 1999; Stone et al., 1999). Genotyping was performed for all siblings and available parents (the majority) in the sample. Semi-automated fluorescent genotyping and data checking and handling were performed using techniques described previously (Fisher et al., 1999).

\section{Quantitative linkage analysis}

Multipoint Variance Components linkage analysis was performed using GENEHUNTER 2.0. (Pratt et al., 2000). Variance Components analysis was performed using a single trait mean, with no QTL or polygenic dominance variance components, and with no manual setting of the mean.

\section{Quantitative association analysis}

The sib-pair quantitative association method of Abecasis et al. (2000) partitions allelic mean effects into orthogonal between-sibship and within-sibship components, and the within-sibship component forms the basis of the association test. The test is therefore analagous to the trañsmission disequilibrium test (Spielman et al., 1993), insofar as it is family based and robust to population stratification. As well as an allelic mean effect, linkage is

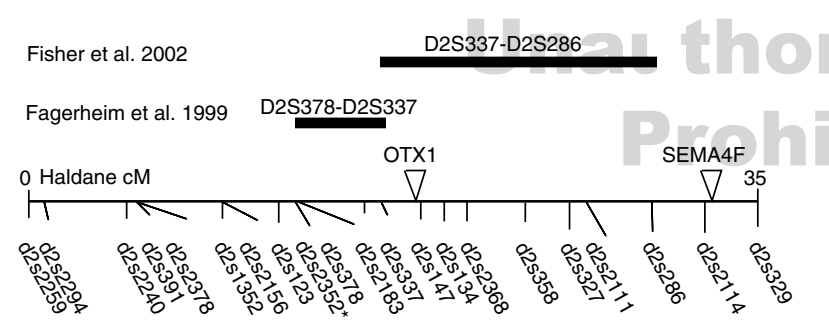

FIGURE 1. Localization of the 2p12-16 locus by dyslexia linkage studies. Localizations correspond to 1-LOD linkage support intervals, and are shown above the markers used in the present study. The positions of OTX1 and Semaphorin4F (SEMA4F) are indicated. Fisher et al. (2002) performed genome-wide linkage analysis in the present study sample, and the present study refined the interval to $12 \mathrm{cM}$. Fagerheim et al. (1999) performed a genome-wide screen of a single extended pedigree (see text for details). * Petryshen et al. (2000) reported preliminary results from a Canadian family sample that included a single-point LOD of 3.00 using quantitative linkage analysis of marker D2S2352 and a measure of spelling disability. simultaneously modelled at the locus using trait variance components, which are conditional on multipoint identity-by-descent (IBD) sharing. For a locus that contributes to trait variance, the linkage variance component is reduced while the allelic association effect is increased (Abecasis et al., 2000). Sib-pair quantitative association analysis was performed using the QTDT suite of applications (Abecasis et al., 2000). GENEHUNTER 2.0 was used to output multipoint IBD data with which to model the variance components, and the programs PRELUDE and FINALE (from the QTDT package) were used for formatting data. Parental genotype data was used in calculating IBD sharing. One thousand transmission permutations were run in order to obtain unbiased empirical significance levels for the association results, and the multi-allele version of the application (QTDT-MULTI) was used for multi-allelic microsatellite markers.

\section{In silico gene characterization}

Starting data in the Genbank database (website: http://www.ncbi.nlm.nih.gov/) on human SEMA4F comprised two sequenced cDNAs (NM_004263.1 and AB021292) obtained from adult hippocampus and forebrain cDNA libraries. Sequence homology searches of the National Center for Biotechnology Information (NCBI) non-redundant database (website: http://www.ncbi.nlm.nih.gov/BLAST) were carried out using both human SEMA4F cDNA clones as probes, and both yielded the same three human genomic sequenced clones with virtually $100 \%$ homology; AC006544, AC006543, and AC007387 (Genbank). SEMA4F maps $982 \mathrm{~kb}$ proximal to D2S2114 according to the draft sequence contig NT_005428 (Genbank), consistent with our own in silico mapping analysis (data not shown). Human SEMA4F has 14 exons (numbered 2-15). Human SEMA4F cDNA clone AB021292 (Genbank) is an alternatively spliced isoform skipping exons 5 and 6 , and causing a frameshift, and human SEMA4F cDNA clone NM_004263.1 (Genbank) is an in-frame alternatively spliced isoform skipping exons 5-8 (not exons 5-9 as recorded in the NCBI Genbank entry). A sequence comparison using BLAST between the human SEMA4F cDNA clone AB021292 and the human genomic clone AC006544 enabled the intronexon structure of human SEMA4F to be constructed for exons 2-4 and 7-15. The rat SEMA4F cDNA clone NM_019272 (Genbank) contained all SEMA4F exons, and sequence homology between this clone and the human genomic clone AC006544 enabled the human sequences of exons 5 and 6 to be determined. 
The resulting in silico full-length human cDNA was verified using the program ORF-FINDER (website: http://www.ncbi.nlm.nih.gov/gorf/gorf.html) to match the predicted open reading frame with that previously described (Encinas et al., 1999).

A published YAC/BAC contig (Resch et al., 1998) of 2p13.3 places OTX1 between the markers D2S147 and D2S2225, and within $2 \mathrm{Mb}$ of D2S147 (Fig. 1). A full-length OTX1 rat cDNA (L32602; Genbank) was used as a BLASTN probe to determine the full-length human cDNA sequence and human intron/exon borders from sequenced human genomic clone AC009501 (Genbank). The resulting sequence was verified against the partial human OTX1 cDNA AB037501 (Genbank). had been previously shown to yield high-quality genotypes. The hybridized PCR products were screened using the WAVE DNA Fragment Analysis System (Taylor et al., 1996), which performs DHPLC with hybridized amplimer duplexes across a range of temperatures. The system can discriminate approximately $90 \%$ of SNPs in fragments of between 200 and 500 nucleotides in length. Any samples showing heteroduplex formation were then sequenced to characterize the polymorphisms responsible. Fluorescence-based dideoxy sequencing was performed using BigDye Terminator sequencing kits (Applied Biosystems, Foster City, California, USA) followed by ABI377 polyacrylamide gel electrophoresis (Applied Biosystems) according to standard protocols.

\section{Exon screening}

Each SEMA4F and OTX1 exon, including up to 90 base pairs of flanking sequence, was screened by denaturing high-performance liquid chromatography (DHPLC) in 30 parents from the sibling sample, followed by nucleotide sequencing of variants. DHPLC is a method for distinguishing heterozygous from homozygous polymerase chain reaction (PCR) amplicon samples (Taylor et al., , 1996). Thirty individuals yield $95 \%$ power to detect a single nucleotide polymorphism (SNP) with a rare allelic frequency of 0.05 , assuming Hardy-Weinberg equilibrium. Exons of SEMA4F and OTX1 were PCR amplified from parental genomic DNA samples that

\section{SNP genotyping}

The SNP SEMSNP1 (Fig. 2) was genotyped by restriction fragment length polymorphism analysis in the entire sample. SEMSNP1 did not cause a known restriction-site change, so a mismatched primer was used for amplification from genomic DNA samples of a 200 base pair region around the SNP, creating an AccI restriction site (GTAGAC) for allele2. The sequences were: allele1 genomic sequence $5^{\prime}$-ATAGAGCTGGCTCCTGGTTGGC-3'; allele2 genomic sequence, 5'-GTAGAGCTGGCTCCTGGTTGGC$3^{\prime}$; mismatched reverse primer, 3'-TCTGGACCGAGGACCAACCG-5' (where bold represents the SNP and underline represents the mismatch) (the

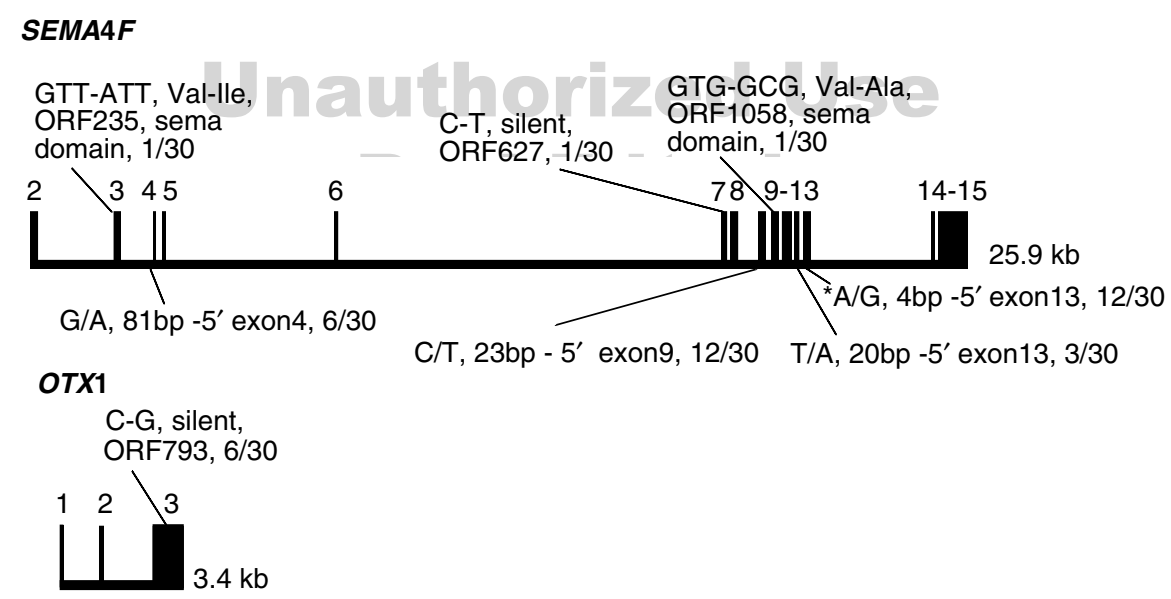

FIGURE 2. Results from intron/exon border characterization, exon screening and single nucleotide polymorphism (SNP) sequencing for Semaphorin4F (SEMA4F) and OTX1. The relative sizes and positions of exons are shown, with exonic SNPs indicated above and intronic SNPs below. Data for each SNP is presented in the order: sequence change (common allele first), amino acid change (if exonic), nucleotide position (in the full-length open reading frame if exonic, or relative to the nearest exon if intronic), protein domain (if exonic and non-synonymous), frequency of heterozygotes in the sample of 30 parents. *This SNP is referred to as SEMSNP1 in the text, and was genotyped in the entire family sample for association analysis. 
forward primer sequence was 5'-TCTTGAAGATCTGGCCTTATTCC-3'). Amplicons were digested with AccI (NEBiolabs, Beverly, Masachusettes, USA), and the resulting products were subject to agarose electrophoresis.

\section{RESULTS}

\section{Quantitative linkage analysis}

Using Variance Components linkage analysis, the 1LOD support interval was refined from approximately $20 \mathrm{cM}$ following the genome-wide screen (Fisher et al., 2002) to $12 \mathrm{cM}$ between markers D2S337 and D2S286 (Figure 1). The peak significance of linkage was not markedly changed from that reported by Fisher et al. (2002) (phoneme awareness $\mathrm{LOD}=2.3$, word recognition $\mathrm{LOD}=1.9$, orthographic coding LOD $=1.7$ ).

\section{Quantitative association analysis}

Results of quantitative sib-pair association analysis are presented in Table 1. Suggestive association was observed for marker D2S2378 (empirical $P=0.004$ for a word-recognition measure, empirical $P=0.004$ for a phonological decoding measure), and marker D2S2114 also showed association with three of the measures (empirical $P<0.05$ ). Unadjusted $P$ values are shown for this analysis (see Conclusions).

Gene characterization and exon screening

We found that the human SEMA4F gene comprises a $2.3 \mathrm{~kb}$ open reading frame distributed over $26.4 \mathrm{~kb}$ of genomic sequence, with 14 exons
(Figure 2). SEMA4F maps $982 \mathrm{~kb}$ proximal to D2S2114 (Figure 1), which was one of the microsatellites to show association. Human OTX1 comprises a $1.065 \mathrm{~kb}$ open reading frame with three exons distributed over $3.4 \mathrm{~kb}$ of genomic sequence (Figure 2). OTX1 maps between the microsatellite markers D2S337 and D2S147 (Figure 1). Figure 2 summarizes the results from the intron/exon border characterization and exon screening of SEMA4F and OTX1. Only two non-synonymous SNPs were found in SEMA4F, each with a heterozygosity of 0.03 , which was too low to perform association analysis. Only one SNP was found in OTX1, which was exonic but silent (6/30 heterozygous).

\section{SNP genotyping and association analysis}

To yield enough power to detect significant familybased association, we decided that a SNP should have heterozygosity $>0.2$ (assessed from the 30 parents) to qualify for genotyping in the whole sample, unless the SNP was one of several different polymorphisms predicted to change the protein. The SNP SEMSNP1 (Figure 2) was an $\mathrm{A} / \mathrm{G}$ change four-nucleotides $-5^{\prime}$ to SEMA4F exon 13 , for which $12 / 30$ individuals were heterozygous $(40 \%)$. Unlike the sequence around this nucleotide, the four-nucleotides $-5^{\prime}$ position is free to vary for eukaryotic exons in general (Stephens and Schneider, 1992), and therefore unlikely to alter RNA splicing. Nonetheless, SEMSNPI was genotyped in the entire sample to test for phenotypic association, since the SNP might have been in linkage disequilibrium with an undetected functional but non-coding polymorphism regulating SEMA4F expression or

TABLE1. Quantitative sib-pair association analysis of microsatellite markers

\begin{tabular}{|c|c|c|c|c|c|}
\hline \multirow[t]{2}{*}{ Marker } & \multirow[t]{2}{*}{$\mathrm{cM}^{\mathrm{a}}$} & \multicolumn{3}{|c|}{ Reading-related measure ${ }^{b}$} & \multirow[b]{2}{*}{ PD } \\
\hline & & WR & PA & $\mathrm{OC}$ & \\
\hline D2S2259 & 0 & 0.088 & & & \\
\hline D2S2240 & 2.4 & & & 0.003 & \\
\hline D2S2378 & 2.9 & 0.004 & & & 0.004 \\
\hline D2S2352 & 14.1 & & 0.061 & & \\
\hline D2S378 & 15.4 & & & & 0.035 \\
\hline D2S337 & 18.6 & & & 0.039 & \\
\hline D2S2111 & 34.5 & & 0.095 & & \\
\hline D2S286 & 36.9 & & & 0.049 & \\
\hline D2S2114 & 38.8 & 0.078 & 0.015 & 0.03 & 0.043 \\
\hline
\end{tabular}

Empirical unadjusted $P$ values $<0.1$ derived from quantitative sib-pair association analysis of 21 microsatellites. Nine markers yielded $P<0.1$. WR, word recognition; PA, phoneme awareness; OC, orthographic choice, PD; phoneme decoding. a Distance from D2S2259 is given in Haldane cM.

'These measures of reading-related abilities have all shown heritabilites $>0.5$ in the Colorado twin study (Olson et al., 1999). The inter-trait correlations were high (range, 0.49-0.84), but twin data has shown that these traits can have independent as well as shared genetic variance (Olson et al., 1999). It is therefore logical to test each measure for association. 
alternative splicing. After genotyping SEMSNP1 in the entire sample, the allelic frequencies were determined to be $0.18: 0.82$, with no deviation from Hardy-Weinberg equilibrium $\left(\chi^{2}=0.036\right)$, yielding heterozygosity $=30 \%$. No significant association was found using quantitative sib-pair analysis of SEMSNP1 with measures of reading disability (all pointwise empirical $P>0.1)$. Note that an intronic SNP $-5^{\prime}$ of exon9 (Figure 2) had a heterozygosity of $40 \%(12 / 30)$ and therefore also met criteria for genotyping. However, the same 12 individuals were heterozygous for this latter SNP as for SEMSNP1. The two SNPs were therefore assumed to be in tight linkage disequilibrium, with the rare alleles of both located on the same chromosomes, and hence equivalent for association analysis.

\section{CONCLUSIONS}

Twenty-one microsatellite markers spanning the 2p12-16 dyslexia QTL were used to refine the region of linkage in our sibling sample from approximately $20 \mathrm{cM}$ to $12 \mathrm{cM}$ between markers D2S337 and D2S286. The positional concordance between our result and that of Fagerheim et al. (1999) (see Figure 1) is encouraging for an etiologically complex trait like dyslexia.

The microsatellites were then tested for quantitative association with four correlated measures of reading disability, and association $P<0.05$ with at least two measures were found for the markers D2S2378 and D2S2114. Bonferroni correction would not be appropriate for analysis using linked markers and correlated phenotypic measures, and might obscure any real signal in samples of this size. However, an inevitable consequence of not making an adjustment is that false-positive associations at $\alpha$ $=0.05$ are likely to be observed. For the 2 p12-16 locus, this can be partly compensated by ignoring $P$ values $<0.05$, which occur for only one phenotypic measure (Table 1), since all of these measures show linkage to some degree at this locus. D2S2378 and D2S2114 are more than $25 \mathrm{cM}$ apart and are not in linkage disequlibrium with one another in this sample (data not shown), and therefore the association results for one marker must represent type I error, assuming that the 2p12-16 linkage is due to only one locus. These association results await confirmation in independent samples.

We decided to move directly to a positional candidate gene screening strategy rather than attempting to saturate the locus with more markers for linkage disequilibrium mapping. Large-scale efforts are currently in progress to identify minimal panels of SNPs that identify common ancestral haplotype blocks separated by recombination hotspots (Johnson et al., 2001). Until such information becomes available, the completion of a pure association-based approach remains impractical for most loci. We determined the genomic structures of candidate genes SEMA4F and OTX1, and the exons of both genes were screened for polymorphisms. However, no coding variants of SEMA4F or OTX1 were identified that could account for the linkage with reading disability observed at $2 \mathrm{p} 12-16$. The possibilities remain that non-coding regulatory variants or major rearrangements at these loci could cause the 2 p12-16 linkage, although our strategy will be to continue screening exons of other candidate genes in the region before investigating these possibilities.

\section{Acknowledgements}

The authors are very grateful to all the families who participated in this study. Thanks to Lorne Lonie and Chris Fletcher for helping with the polymorphism screening, Goncalo Abecasis for advice on using QTDT, and Laurence MacPhie for helpful comments on the manuscript. Sponsorship: C.F. was a Wellcome Trust Prize Student. A.P.M. is a Wellcome Trust Principal Research Fellow. The Colorado Twin Study was supported in part by program project and center grants from NICHD (HD-11681 and HD27802). All molecular work was funded by the Wellcome Trust.

\section{REFERENCES}

Abecasis GR, Cardon LR, Cookson WOC (2000). A general test of association for quantitative traits in nuclear families. Am J Hum Genet 66:279-292.

Acampora D, Mazan S, Avantaggiato V, Barone P, Tuorto F, Lallemand Y, et al. (1996). Epilepsy and brain abnormalities in mice lacking the Otx1 gene. Nat Genet 14:218-222.

Acampora D, Mazan S, Tuorto F, Avantaggiato V, Trembley JJ, Lazzaro D, et al. (1998). Transient dwarfism and hypogonadism in mice lacking Otx 1 reveal prepuscent stage-specific control of pituitary levels of GH, FSH and LH. Development 125:1229-1239.

Acampora D, Barone P, Simeone A (1999). Otx genes in corticogenesis and brain development. Cerebral Cortex 9:533-542.

Cardon LR, Smith SD, Fulker DW, Kimberling WJ, Pennington BF, DeFries JC (1994). Quantitative trait locus for reading disability on chromosome 6. Science 266:276-279.

Cardon LR, Smith SD, Fulker DW, Kimberling WJ, Pennington BF, DeFries JC (1995). Quantitative trait 
locus for reading disability: correction. Science 268: 1553 .

DeFries JC, Fulker DW, LaBuda MC (1987). Evidence for a genetic aetiology in reading disability of twins. Nature 329:537-539.

Eden GF, VanMeter JW, Rumsey JM, Maisog JM, Woods RP, Zeffiro TA (1996). Abnormal processing of visual motion in dyslexia revealed by functional brain imaging. Nature 382:66-69.

Encinas JA, Kikuchi K, Chedotal A, de Castro F, Goodman CS, Kimura T (1999). Cloning, expression, and genetic mapping of Sema $\mathrm{W}$, a member of the semaphorin family. Proc Natl Acad Sci USA 96:24912496.

Fagerheim T, Raeymaekers P, Tonnessen FE, Pedersen M, Tranebjaerg L, Lubs HA (1999). A new gene (DYX3) for dyslexia is located on chromosome 2. $\mathrm{J} \mathrm{Med}$ Genet 36:664-669.

Fisher SE, Smith SD (2001). Progress towards the identification of genes influencing developmental dyslexia. In: Fawcett AJ, editor. Dyslexia: theory and good practice. London: Whurr. pp. 39-64.

Fisher SE, Marlow AJ, Lamb J, Maestrini E, Williams DF, Richardson AJ, et al. (1999). A quantitative trait locus on chromosome $6 \mathrm{p}$ influences different aspects of developmental dyslexia. Am J Hum Genet 64:146-156.

Fisher SE, Francks C, Marlow AJ, MacPhie IL, Newbury DF, Cardon LR, et al. (2002). Independent genome-wide scans identify a chromosome 18 quantitative-trait locus influencing dyslexia. Nat Genet. 30:86-91.

Hartl DL, Clark AG (2001). Principles of population genetics. Sunderland, MA: Sinauer Associates.

Johnson GCL, Esposito L, Barratt BJ, Smith AN, Heward J, Di Genova G, et al. (2001). Haplotype tagging for the identification of common disease genes. Nat Genet 29:233-237.

Kirschner LS, Taymans SE, Pack S, Pak E, Pike BL, Chandrasekharappa SC, et al. (1999). Genomic mapping of chromosomal region 2p15-p21 (D2S378D2S391): Integration of Genemap'98 within a framework of Yeast and Bacterial Artificial Chromosomes. Genomics 62:21-33.

Kolodkin A, Matthes DJ, Goodman CS, (1993). The semaphorin genes encode a family of transmembrane and secreted growth cone guidance molecules. Cell 75:1389-1399.

Marlow AJ, Fisher SE, Richardson AJ, Francks C, Talcott JB, Monaco AP, et al. (2001). Investigation of quantitative measures related to reading disability in a large sample of sib-pairs from the UK. Behav Genet 31:219-230.

Nakamura F, Kalb RG, Strittmatter SM, (2000). Molecular basis of semaphorin-mediated axon guidance. J Neurobiol 44:219-229.

Nopola-Hemmi J, Myllyluoma B, Haltia T, Taipale M, Ollikainen V, Ahonen T, et al. (2001). A dominant gene for developmental dyslexia on chromosome 3. J Med Genet 38:658-664.

Olson RK, Datta H, Gayán J, DeFries JC (1999). A behavioral-genetic analysis of reading disabilities and component processes. In: Klein RM, McMullen PA, editors. Converging methods for understanding reading and dyslexia. Cambridge: MIT Press. pp. 133-151.

Petryshen TL, Kaplan BL, Hughes ML, Field LL, (2000). Evidence for the chromosome 2p15-p16 dyslexia susceptibility locus (DYX3) in a large Canadian data set [abstract]. Am J Med Genet 96:472.

Pratt SC, Daly MJ, Kruglyak L, (2000). Exact multipoint quantitative-trait linkage analysis in pedigrees by variance components. Am J Hum Genet 66:1153-1157.

Resch K, Korthaus D, Wedemeyer N, Lengeling A, Ronsiek M, Thiel C, et al. (1998). Homology between human chromosome 2 p13.3 and the wobbler critical region on mouse chromosome 11: comparative highresolution mapping of STS and EST loci on YAC/BAC contigs. Mamm Genome 9:893-898.

Smith SD, Gilger JW, Pennington BF (1996). Dyslexia and other specific learning disorders. In: Rimoin DL, Connor JM, Pyeritz RE, editors. Principles and practice of medical genetics. New York: Churchill Livingston. pp. $1767-1789$.

Spielman RS, McGinnis RE, Ewens WJ (1993). Transmission test for linkage disequilibrium: the insulin gene region and insulin-dependent diabetes mellitus (IDDM). Am J Hum Genet 52:506-516.

Stephens RM, Schneider TD, (1992). Features of spliceosome evolution and function inferred from an analysis of the information at human splice sites. $J \mathrm{Mol}$ Biol 228:1124-1136.

Stone E, Lotery AJ, Munier FL, Heon E, Piguet B, Guymer RH, et al. (1999). A single EFEMP1 mutation associated with both Malattia Leventinese and Doyne honeycomb retinal dystrophy. Nat Genet 22:199-202.

Taylor P, Munson K, Gjorde D (1996). Detection of mutations and polymorphisms on the WAVE ${ }^{\mathrm{TM}}$ DNA fragment analysis system. Transgenomic Inc., Omaha, NE, USA. Application Note No. 101. 